\title{
Fever and 'negative' blood cultures in an elderly woman
}

\author{
J Clive Graham, Nick Kennedy, Fred J Nye
}

Regional Infectious

Diseases Unit,

Fazakerley Hospital,

Lower Lane,

Liverpool L9 7AL,

UK

JC Graham

N Kennedy

FJ Nye

Correspondence to

JC Graham, Department

of Microbiology, Royal

of Microbiology, Royal
Victoria Infirmary, Queen

Victoria Infirmary, Queen

Upon Tyne NE1 4LP, UK

Accepted 25 September
1996

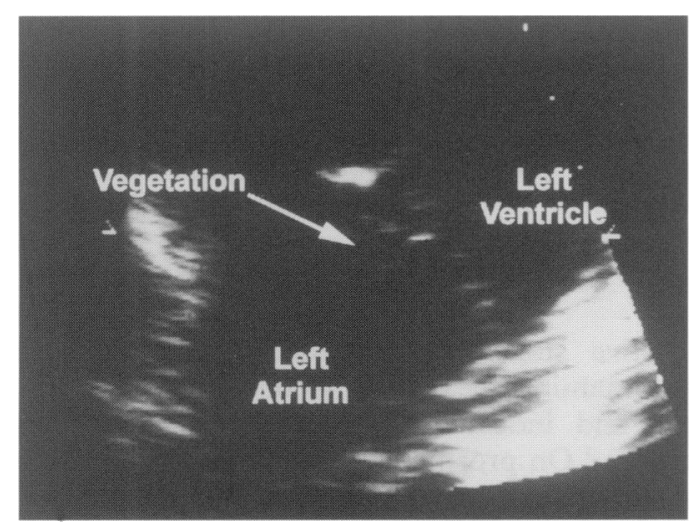

\section{Questions}

1 What is the diagnosis?

2 Which criteria can be used to help make this diagnosis?

3 Where are coagulase-negative staphylococci normally found and in what clinical situations are they well-recognised as pathogenic organisms?

Figure Transthoracic echocardiography 
Answers

QUESTION 1

Bacterial endocarditis due to coagulase-negative staphylococci affecting the mitral valve.

\section{QUESTION 2}

Coagulase-negative staphylococci are skin commensals and frequently contaminate blood cultures. To distinguish true bacteraemia from contamination, two or more blood cultures taken on separate occasions should contain the same strain. The isolates should be of the same species and have identical antibiograms; if necessary, they can be sent to a reference laboratory for typing: if they are shown to be truly identical the diagnosis will be strengthened.

The distinction between endocarditis and uncomplicated bacteraemia caused by coagulase-negative staphylococci depends upon the interpretation of clinical, microbiological and echocardiographic data. Scoring systems such as the 'Duke' criteria have been developed to increase diagnostic accuracy. ${ }^{1}$

\section{QUESTION 3}

Coagulase-negative staphylococci are part of the normal flora of the skin and mucous membranes. In specific clinical situations they are well recognised as pathogens (eg, in neonates, the immunocompromised and those with indwelling prosthetic devices such as heart valves and joint prostheses). ${ }^{2}$ Endocarditis is usually caused by $S$ epidermidis. Other specific coagulase-negative staphylococci such as $S$ saprophyticus are a common cause of urinary tract infection but are rarely responsible for more invasive disease.

\section{Discussion}

Historically coagulase-negative staphylococci have been regarded as contaminants when isolated in blood cultures and indeed only $6.3 \%$ reflect true bacteraemias. ${ }^{3}$ On prosthetic valves they are the commonest cause of endocarditis, especially in the first postoperative year. On native valves coagulase-negative staphylococci represent between $1 \%$ and $10.4 \%$ of bacterial isolates and there is evidence to suggest that the incidence may be increasing. The onset of the disease is insidious with low grade fever, weakness, anorexia and weight loss, although more acute presentation may occur in up to $26 \%$ of cases. Threequarters of the patients have pre-existing cardiac abnormalities, the most common being mitral valve prolapse. ${ }^{4}$

1 Bayer AS, Ward JI, Ginzton LE, Shapiro SM. Evaluation of new clinical criteria for the diagnosis of infective endocarditis. Am F Med 1994; 96: 211 -9.

2 Bailey EM, Constance TD, Albrecht LM, Rybak MJ Coagulase-negative Staphylococci: incidence, pathogenicity and treatment in the 1990s. Ann Pharmacother 1990; 24: 714-20.

3 Arber N, Militanu A, Ben-Yehuda A, Krivoy N, Pinkhas J, Sidi Y. Native valve Staphylococcus epidermidis endocarditis: report of seven cases and review of the literature. $\mathrm{Am} \mathrm{f} \mathrm{Med}$ 1991; 90: $758-61$.

\section{Learning points}

- coagulase negative staphylococci are frequently isolated from blood cultures

- most are contaminants from normal skin flora

- they infect a wide range of prosthetic devices and are a not infrequent cause of native valve endocarditis

- native valve endocarditis caused by coagulasenegative staphylococci tends to present insidiously and a high index of suspicion is required to make the diagnosis

When clinical suspicion is high, multiple blood cultures should be taken as although growth of coagulase-negative staphylococci is not invariable, even in bacterial endocarditis, continued isolation of coagulase-negative staphylococci increases the likelihood of true bacteraemia. ${ }^{5}$ Antimicrobial chemotherapy should be guided by sensitivity testing. Hospital-acquired isolates tend to be multiply resistant and a glycopeptide such as vancomycin or teicoplanin should be used, preferably in combination with a second agent. Despite antibiotic treatment, valve replacement may be necessary in around $50 \%$ of cases. Mortality rates of up to $36 \%$ have been reported, even with aggressive medical and surgical management. ${ }^{4}$

Although we were unable to identify a skin or mucosal source we suspect that an intravenous cannula site may have produced a transient bacteraemia with secondary infection of a prolapsing mitral valve. Certain virulence factors have been determined among coagulase-negative staphylococci. One of the most widely studied is 'slime' production which protects the organism from the host defence system and antimicrobials.

In summary, the diagnosis of native valve endocarditis due to coagulase-negative staphylococci requires a high index of suspicion by the clinician as the onset is insidious with few systemic signs. Multiple blood cultures should be performed and the results of these carefully interpreted in conjunction with other clinical and laboratory findings (box).

\section{Final diagnosis}

Bacterial endocarditis caused by coagulasenegative staphylococci affecting the mitral valve.

Keywords: coagulase-negative staphylococci, bacterial endocarditis, native valve

4 Etienne J, Eykyn SJ. Increase in native valve endocarditis caused by coagulase negative staphylococci: an AngloFrench clinical and microbiological study. Br Heart 1990; 64: $381-4$

5 Peacock SJ, Bowler ICJW, Crook DWM. Positive predictive value of blood cultures growing coagulase-negative staphylococci (letter). Lancet 1995; 346: 191-2. 